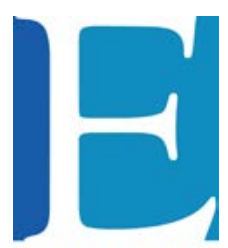

EVALUAR
2017, Vol. 17, No. 1

ISSN $1667-4545$

Recuperado de www.revistas.unc.edu.ar

Laboratorio de Evaluación Psicológica y Educativa

Facultad de Psicología - Universidad Nacional de Córdoba

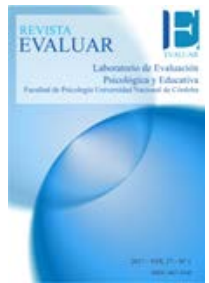

\title{
Dimensionalidad de un Test de Conducta Prosocial
}

\author{
Sofía Esmeralda Auné * 1 , Horacio Félix Attorresi ${ }^{2}$ \\ 1. Instituto de Investigaciones de la Facultad de Psicología de la Universidad de Buenos Aires, CONICET, Argentina. \\ 2. Instituto de Investigaciones de la Facultad de Psicología de la Universidad de Buenos Aires, Argentina.
}

Introducción

Método

Resultados

Discusión

Referencias

Recibido: 09/11/2016 Revisado: 12/12/2016 Aceptado: 23/12/2016

\section{Resumen}

La conducta prosocial es toda conducta social positiva con o sin motivación altruista. La realización de este tipo de conductas eleva el sentimiento de autoestima y contribuye a la formación de redes de apoyo. El objetivo de este trabajo es estudiar la dimensionalidad de la Escala de Conducta Prosocial (ECP) de Auné, Abal y Attorresi. La misma se encuentra conformada por 28 ítems. Se seleccionó una muestra por accesibilidad de 692 individuos de Gran Buenos Aires e interior de la Argentina, quienes respondieron la ECP. Se eliminaron 25 casos considerados anómalos según la distancia de Mahalanobis. El estudio de la dimensionalidad se realizó mediante la técnica de Análisis Factorial Exploratorio basado en la matriz de correlaciones policóricas con rotación promax. Se eligió el procedimiento Análisis Paralelo para determinar el número de factores. Éstos fueron estimados mediante el método de mínimos cuadrados no ponderados. Un modelo de 15 ítems y dos factores logró índices de ajuste adecuados $(\mathrm{GFI}=.99, \mathrm{RMSR}=.0384)$, explicando el $50.61 \%$ de la varianza. Los resultados concuerdan con la bibliografía existente, pues uno de los factores agrupó ítems relacionados con confortar mientras que el segundo factor reunió ítems que implican ayuda.

Palabras clave: conducta prosocial, dimensionalidad, ayuda, confortar, psicología positiva

\begin{abstract}
Prosocial behavior is regarded as positive social conduct with or without altruistic motivation. The realization of this type of behavior boosts self-esteem and contributes to the construction of support networks. The aim of this study was to analyze the dimensionality of the Prosocial Behavior Scale (ECP) by Aune, Abal, and Attorresi, comprising 28 items. An accidental sample of 692 individuals who responded to the ECP was selected from Gran Buenos Aires and Argentine provinces. Twenty-five cases were disregarded, as they were considered anomalous according to Mahalanobis distance. The study was carried out using Exploratory Factor Analysis based on a polychoric correlation matrix with promax rotation. Parallel Analysis procedure was chosen to determine the number of factors, which was estimated by the method of non-weighted least squares. A model of 15 items and two factors achieved adequate fit indices (GFI $=.99$, RMSR $=.0384$ ), accounting for $50.61 \%$ of the variance. These results are consistent with the literature, since items related to comfort were grouped by one of the factors, while items involving aid were grouped by the second factor analyzed.
\end{abstract}

Keywords: prosocial behavior, dimensionality, aid, comforting, positive psychology 


\section{Introducción}

Las conductas prosociales generalmente son definidas como actos voluntarios orientados a beneficiar a otras personas (Caprara, Steca, Zelli, \& Capanna, 2005). Parece haber poco consenso sobre una definición común de comportamiento prosocial y sobre cuáles son las diferencias entre los comportamientos prosociales y los altruistas (Auné, Blum, Abal, Lozzia, \& Attorresi, 2014; Lemos \& Richaud de Minzi, 2014). Para Roche-Olivar (1998) la conducta prosocial es únicamente aquella acción que favorece a otra persona, grupo o meta social, aumentando consecuentemente la probabilidad de generar una reciprocidad positiva, de calidad y solidaria en las relaciones interpersonales o sociales. La conducta prosocial también salvaguarda la identidad, creatividad e iniciativa de las personas o grupos implicados. En cambio, para González-Portal (2000) el comportamiento prosocial es simplemente toda conducta social positiva con o sin motivación altruista.

Es así que, mientras que para varios autores la conducta es considerada prosocial únicamente cuando es motivada desde el altruismo, otros consideran que un acto prosocial puede estar orientado por el egoísmo, así como por ideales morales o la intención de favorecer al propio grupo (Batson \& Powell, 2003). Los motivos del comportamiento prosocial pueden ser incluso inespecíficos o desconocidos (Beilin, 2013). Es, sin duda, un fenómeno complejo por su ambivalencia emocional y actitudinal; y refleja la tensión entre valores sociales y necesidades defensivas (Hirschberger, 2006). Para Carlo y Randall (2001), en el análisis de la conducta prosocial deberían examinarse los contextos interpersonales en los que se realiza.

Se ha denominado prosocialidad a la tendencia de la personalidad a realizar acciones solidarias (Caprara, Alessandri, Di Giunta, Panerai, \& Eisenberg, 2010). Mientras que el término conducta prosocial hace referencia a la acción en sí, la prosocialidad sería la dimensión latente común que subyace a dichos comportamientos. Esta dimensión varía entre los individuos. El control emocional, la capacidad de toma de perspectiva, la confianza en la capacidad para ayudar a otros y los valores de autotrascendencia favorecen la prosocialidad (Alessandri, Caprara, Eisenberg, \& Steca, 2009).

Realizar frecuentemente conductas prosociales se relaciona con una mayor estabilidad emocional, autoestima, confianza, rendimiento académico y otras variables salugénicas (Caprara, Alessandri, \& Eisenberg, 2012). De hecho, la conducta prosocial es un tema del campo de la Psicología Positiva (Castro-Solano, 2010). En cambio, una baja frecuencia de conductas prosociales se asocia con el comportamiento agresivo y los problemas afectivos (Scourfield, John, Martin, \& McGuffin, 2004).

Una diversidad de factores contribuyen a la realización de la conducta prosocial: tanto las características de la situación que se presenta, como variables culturales o individuales tales como la edad, género, nivel socioeconómico y características afectivas y cognitivas. Ninguna variable, por sí sola, explica totalmente este tipo de comportamientos (Batson \& Powell, 2003).

Es en el proceso de socialización donde se aprende a actuar en beneficio de otra persona (Garaigordobil \& Berrueco, 2007). Determinadas conductas paternas, tales como expectativas y patrones conductuales, contribuyen a que posteriormente los hijos puedan realizar conductas prosociales. Estas conductas favorecedoras de la acción prosocial son la aceptación parental, la toma de perspectiva y la preocupación empática de los padres (Richaud de Minzi, Lemos, \& Mesurado, 2011).

El constructo conducta prosocial ha sido concebido clásicamente como multidimensional. Las primeras tipologías distinguían un gran número de dimensiones (p. ej.: Roche-Olivar, 1998), pero, en los últimos años, la bibliografía propone un número más reducido de factores 
generales. Además, se incorpora a la empatía como parte del comportamiento prosocial y no sólo como moduladora del mismo (Caprara, Steca, et al., 2005). Dunfield (2014) distingue entre ayudar, compartir y confortar. La autora plantea que ayudar alivia la necesidad instrumental del beneficiario y compartir intenta satisfacer el deseo material de otra persona, mientras que confortar se orienta a aplacar el dolor emocional. Warneken y Tomasello (2009) proponen como factores confortar, compartir, informar y ayudar instrumentalmente. Hay y Cook (2007) delimitan sentimientos por el otro, trabajar con otro y atender a otro. Caprara, Steca, et al. (2005) distinguen entre compartir, cuidar, ayudar y ser empático.

En relación con la evaluación de la conducta prosocial, una gran proporción de los instrumentos existentes se destina a niños y adolescentes. Los orientados a la adultez y vejez resultan escasos, y la realización de estudios acerca de las propiedades psicométricas de dichos tests es un tópico poco desarrollado (Auné et al., 2014). Existen conductas prosociales que son específicas de la adultez, por ejemplo la donación voluntaria de sangre o desempeñarse en una profesión de ayuda aún poniendo en riesgo el bienestar personal.

\section{Objetivo}

Dada la escasez de instrumentos dedicados a la medición de la conducta prosocial en la etapa adulta, se han redactado ítems para conformar una Escala de Conducta Prosocial (ECP) para adultos. Los objetivos del presente trabajo son explorar la estructura factorial de la Escala de Conducta Prosocial (ECP) de Auné, Abal y Attorresi (2016a) para residentes de Gran Buenos Aires e interior de la Argentina y determinar el número de factores más adecuado para dicha escala. Es decir, estos objetivos corresponden al comienzo de la construcción del instrumento.

\section{Metodología}

Participantes

Participaron 692 individuos (65\% mujeres, Media $=27$ años, $\mathrm{DE}=6.26$ ) seleccionados por accesibilidad, residentes tanto de Gran Buenos Aires (25\%) como del interior de la Argentina (75\%). El 13\% contaba con un nivel educativo de hasta secundario incompleto y el $24 \%$, con un nivel de secundario completo sin terciario. El 63\% restante tenía un nivel de terciario incompleto como mínimo.

\section{Instrumento}

Escala de Conducta Prosocial (ECP, Auné et al., 2016a). Está compuesta por 28 ítems, con seis opciones de respuesta tipo Likert (Nunca, Casi nunca, A veces, Con frecuencia, Casi siempre, Siempre). Los reactivos muestran distintos grados de beneficio hacia el otro, así como variados beneficiarios de la acción.

\section{Procedimiento}

En relación al análisis de datos anómalos multivariados, se calculó la distancia de Mahalanobis utilizando un punto de corte de $p \leq .001$ (Tabachnick \& Fidell, 2001). En forma progresiva, para evitar efectos de solapamiento e inundación/empantanamiento, se eliminaron de a uno 25 casos de la muestra, quedando un nuevo tamaño de 667 participantes.

Los valores del índice de Mardia (1970) para la asimetría y curtosis multivariante obtenidos con el macro para SPSS de DeCarlo (1997; coeficiente de asimetría de 57.21, $\chi^{2}$ ${ }_{[680]}=6350, p<.001$; coeficiente de curtosis de 195.839, $p<.001$ ) mostraron que los datos no se distribuían de forma normal multivariante. Cuando la distribución no es normal existe una tendencia del estadístico $\chi^{2}$ a rechazar la hipóte- 
sis nula (Bearden, Sharma, \& Teel, 1982). Por lo tanto, el ajuste del modelo se evaluó en base a indicadores que no dependen directamente de $\chi^{2}$ (Ferrando \& Lorenzo-Seva, 2014), el índice de bondad de ajuste (Goodness of Fit Index, GFI) y la raíz media cuadrática residual (Root Mean Square Residual, RMSR).

Se utilizaron la medida de adecuación muestral de Kaiser-Meyer-Olkin (KMO) y la prueba de esfericidad de Bartlett para determinar si el análisis factorial exploratorio (AFE) era adecuado. Una vez verificada dicha adecuación $\left(\mathrm{KMO}=.91\right.$; Bartlett: $\left.\chi_{[378]}^{2}=6807 ; p<.001\right)$ se procedió a realizar el AFE mediante el programa Factor 9.2. (Lorenzo-Seva \& Ferrando, 2013) considerando una estructura unifactorial.

Actualmente se afirma que cuando la validez de un constructo es analizada con datos ordinales provenientes de escalas Likert, los factores resultantes muestran un mejor ajuste al modelo teórico si la factorización es realizada con la matriz de correlaciones policóricas en vez de emplear la de Pearson (Holgado-Te1lo, Chacón-Moscoso, Barbero-García, \& Vila-Abad, 2010). La utilización de la correlación de Pearson implica suponer distribución normal y un nivel de medición de la variable, como mínimo, intervalar.

El hallazgo de correlaciones interfactor mayores a .32 motivó la utilización del método promax para la rotación (Tabachnick \& Fidell, 2001) con un parámetro kappa de 4 (Hendrickson \& White, 1964). Esta rotación oblicua no asume incorrelación entre los factores y es por ello que resulta adecuada para estos casos.

El método de extracción de factores fue el de mínimos cuadrados no ponderados (Unweighted Least Squares, ULS) que según las recomendaciones actuales, resulta más adecuado que el de máxima verosimilitud cuando la factorización de reactivos se realiza a través de la matriz de correlaciones policóricas (Lloret-Segura et al., 2014).
Se utilizó el análisis paralelo (Horn, 1965) para determinar el número de factores. Dicho método posee cierta robustez frente a desviaciones de la normalidad (Buja \& Eyuboglu, 1992), especialmente cuando se basa en la matriz de correlaciones policóricas (Garrido, Abad, \& Ponsoda, 2013), como en este caso.

\section{Resultados}

La unidimensionalidad no pudo sostenerse, pues el primer factor sólo explicaba el 32\% de la varianza, un valor menor al $40 \%$ considerado por Carmines y Zeller (1979) para sostener una sola dimensión. Además, la razón entre el primer autovalor y el segundo era inferior a 5 (3.46), lo cual según Martínez-Arias, Hernández-Lloreda y Hernández-Lloreda (2006) indica que no hay un factor claramente predominante. Los indicadores GFI $=.94(<.95 ; \mathrm{Hu} \&$ Bentler, 1999) y RMSR $=.0925$ ( $>.0387$, no satisface el criterio de Kelley) señalaron que el ajuste unifactorial era pobre (Lloret-Segura, Ferreres-Traver, Hernández-Baeza, \& Tomás-Marco, 2014). Por las razones expuestas se resolvió realizar un nuevo AFE imponiendo una estructura bifactorial.

En sucesivos análisis se eliminaron los reactivos asociados a los mayores residuos estandarizados, hasta que el RMSR satisfizo el criterio de Kelley. Según Kelley (1935) si RMSR no excede $1 / \sqrt{\mathrm{n}}$, donde $n$ es el tamaño de muestra, puede considerarse que el ajuste del modelo es aceptable. En este trabajo el tamaño de muestra es 667 , por lo tanto se consideró un buen ajuste ya que el RMSR es .0384 , menor que $.0387=$ $1 / \sqrt{667}$. Una vez que el modelo ajustó, se conservaron los ítems con cargas factoriales mayores que .30 , y que en cada dimensión cumpliesen el criterio de la Teoría Clásica de los Tests de no aumentar el coeficiente alfa de Cronbach al quitar el reactivo. 
Un modelo de 15 ítems y dos factores logró índices de ajuste adecuados (RMSR = .0384 , Cr. Kelley <.0387; GFI $=.99$, mayor que .95; Hu \& Bentler, 1999), explicando el 50.61\% de la varianza. El primer factor $\left(1^{\circ} \mathrm{F}\right)$, compuesto por 7 ítems $(5,6,8,20,25,26,28)$ obtuvo un coeficiente alfa de Cronbach de .77 mientras que el segundo factor $\left(2^{\circ} \mathrm{F}\right)$, fue conformado por 8 ítems $(1,4,9,10,13,19,22,23)$, con un coeficiente alfa de Cronbach de .85 . Utilizando el criterio de DeVellis (2003), la confiabilidad del primer factor se interpreta como aceptable y la del segundo factor como muy buena. La correlación entre los factores fue de .49 .

La Tabla 1 muestra las matrices de configuración y de estructura del modelo de 15 ítems. Son dos matrices rotadas. Las correlacio- nes de cada ítem con cada factor se muestran en la matriz de estructura, mientras que en la matriz de configuración se observan coeficientes análogos a los coeficientes beta del análisis de regresión múltiple. Los coeficientes de la matriz de configuración indican la importancia relativa de cada factor para explicar el puntaje individual de cada ítem (Pérez \& Medrano, 2014). Como se puede observar en dicha tabla, interpretando ambas matrices, tanto las cargas factoriales como las correlaciones fueron elevadas, oscilando entre .40 y .89 en la dimensión correspondiente al ítem. En consecuencia, se puede inferir un ajuste adecuado de la escala al modelo bifactorial (Vélez \& Martínez-Lugo, 1995).

\section{Tabla 1}

Matrices de Configuración y de Estructura.

\begin{tabular}{|c|c|c|c|c|c|}
\hline \multirow{2}{*}{\multicolumn{2}{|c|}{ Ítems }} & \multicolumn{2}{|c|}{$\begin{array}{c}\text { Matriz de } \\
\text { configuración }\end{array}$} & \multicolumn{2}{|c|}{ Matriz de estructura } \\
\hline & & $\mathbf{1}^{\circ} \mathrm{F}$ & $2^{\circ} \mathrm{F}$ & $1^{\circ} \mathrm{F}$ & $2^{\circ} \mathrm{F}$ \\
\hline 1 & Relego mi beneficio personal para ayudar a otros. & .17 & .40 & .37 & .48 \\
\hline 4 & Realizo actividades como voluntario. & -.20 & .89 & .23 & .78 \\
\hline 5 & Cuando siento que alguien está mal le demuestro que lo entiendo. & .66 & .00 & .66 & .32 \\
\hline 6 & Actúo como bastón de los demás. & .59 & .05 & 62 & .34 \\
\hline 8 & Siento todo dolor ajeno como propio. & .47 & .21 & .58 & .44 \\
\hline 9 & Me comprometo con causas nobles. & .17 & .68 & .50 & .76 \\
\hline 10 & Dedico un tiempo importante de mi vida a mejorar el mundo. & .02 & .68 & .35 & .69 \\
\hline 13 & Me ocupo del bienestar de cualquier individuo, grupo o comunidad. & .12 & .61 & .42 & .67 \\
\hline 19 & Me quedo con lo justo y necesario para vivir y reparto todo lo demás. & .18 & .51 & .43 & .60 \\
\hline 20 & Me pongo en el lugar del otro. & .64 & .10 & .68 & .41 \\
\hline 22 & Participo en actividades solidarias. & -.09 & .72 & .26 & .67 \\
\hline 23 & Hago donaciones a organizaciones benéficas. & -.09 & .76 & .28 & .72 \\
\hline 25 & $\begin{array}{l}\text { Si una persona me cuenta un conflicto, intento que comprenda el punto } \\
\text { de vista de la otra parte. }\end{array}$ & .60 & -.09 & .56 & .21 \\
\hline 26 & Intento "levantar" la autoestima a mis amigos. & .81 & -.15 & .74 & .25 \\
\hline 28 & Aconsejo a conocidos sobre trabajo. & .41 & .06 & .44 & .26 \\
\hline
\end{tabular}




\section{Discusión}

El modelo bifactorial se consideró adecuado ya que explicó más del $50 \%$ de la varianza y porque la discrepancia entre la matriz de varianzas y covarianzas observada y la reproducida por el modelo fue baja (RMSR), cumpliendo la condición de Kelley. También el GFI indicó un ajuste satisfactorio. El análisis paralelo indicó, asimismo, que la estructura bifactorial era la más apropiada.

La interpretación de cada uno de los dos factores de este modelo se realizó observando las correlaciones ítem-factor más elevadas (Glutting, Monaghan, Adams, \& Sheslow, 2002). Los resultados son coherentes con la bibliografía existente, en la medida en que el primer factor agrupó ítems relacionados con lo que se denominó confortar al otro, por ejemplo "me pongo en el lugar del otro", mientras que el segundo factor reunió ítems que implican ayuda y esa fue su denominación, por ejemplo "realizo actividades como voluntario". Resulta importante la diferenciación entre los distintos tipos de comportamiento prosocial ya que la ausencia de esta diferenciación ha resultado en inconsistencias en las discusiones teóricas. Se han encontrado, incluso, distintas rutas de desarrollo y correlatos para diferentes tipos de acción prosocial (Dunfield, Kuhlmeier, O'Connell, \& Kelley, 2011). Los trabajos realizados hasta el momento, si bien con otros instrumentos de medición de la conducta prosocial, encontraron una estructura como mínimo trifactorial. Por ejemplo, con la Escala para la Medición de la Conducta Prosocial en Adultos (Prosocialness Scale for Adults, PSA) de Caprara, Steca, et al. (2005), como ya se indicó, se encontraron cuatro factores: compartir, cuidar, ayudar y ser empático. En este trabajo, realizado con la ECP, la dimensión compartir no se distinguió de la dimensión ayuda, sino que presentó una diferenciación bifactorial básica entre los comportamientos que suponen un apoyo emocional y aquellos que representan una asistencia de tipo instrumental a otro. A su vez, no se incluyeron ítems correspondientes a la dimensión cuidar propuesta por Caprara, Steca, et al. (2005).

La dimensión confortar incluye comportamientos de apoyo emocional. Estos comportamientos se desarrollan alrededor del segundo año de vida y tienen como base la posibilidad de distinguir entre el sí mismo y los otros (Decety \& Meyer, 2008) y la capacidad de regular las propias emociones negativas (Paulus, Kühn-Popp, Licata, Sodian, \& Meinhardt, 2013). Esta faceta se corresponde con la dimensión confortar de Dunfield (2014) y de Warneken y Tomasello (2009). Hay y Cook (2007) la describen como sentimientos por el otro y Caprara, Steca, et al. (2005) la denominan ser empático. Es decir, esta dimensión ha sido observada reiteradamente (Auné, Abal, \& Attorresi, 2016b).

Como se ha mencionado, Caprara, Steca, et al. (2005) destacan la empatía como dimensión de la conducta prosocial en la adultez y proponen incluirla en las escalas para este ciclo vital. Para ellos, la capacidad de sentir las necesidades y estados de ánimo ajenos sería una parte integral de la conducta prosocial (Caprara, Capanna, Steca, \& Paciello, 2005). Aunque es un tópico controvertido si la empatía en sí es parte de la prosocialidad (Auné et al., 2016b), existe un acuerdo generalizado acerca de que la acción de confortar sí lo es.

La dimensión ayuda incluye tanto comportamientos solidarios clásicos como una orientación general a dar, por ejemplo dinero, bienes o tiempo para mejorar la situación de otro. El comportamiento de ayuda se comienza a observar ya en niños de un año y medio (Warneken \& Tomasello, 2006).

Tanto confortar como ayudar son conductas positivas hacia un otro. Sin embargo, la realización de conductas incluidas en la dimensión ayuda requiere, en general, un nivel de esfuerzo más alto que aquellas incluidas en la dimensión confortar. Además, hallazgos recientes realiza- 
dos mediante electroencefalograma indican que ambos comportamientos se sustentan en bases neurofisiológicas específicas y diferentes, con patrones separados de activación cerebral al realizar cada uno de ellos (Paulus et al., 2013). Mientras que confortar se vinculó con el lóbulo frontal izquierdo, un marcador de proceso emocional; la ayuda se relacionó con el lóbulo temporal, probablemente reflejando la comprensión relacionada con objetivos. Estos resultados son coherentes con la correlación moderada observada entre los factores. Por lo cual, se concluye que se ha encontrado una estructura factorial adecuada para la ECP.

En relación a las limitaciones del presente estudio, al ser la ECP un autoinforme, el nivel real de rasgo latente (conducta prosocial) estimado por el puntaje total incluye un componente subjetivo, y puede estar sesgado. En segundo lugar se pueden señalar las características de la muestra. Futuras investigaciones podrán dilucidar si estos resultados se mantienen en otras regiones de la Argentina, lo cual permitirá generalizar los resultados a una población más amplia.

Por otra parte, se espera modelizar la ECP con modelos de la Teoría de la Respuesta al Ítem (TRI), lo cual permitirá caracterizar cada reactivo en base a sus propios parámetros, y construir un instrumento con propiedades invariantes entre poblaciones. Los modelos de la TRI han sido poco usados para tests de comportamiento típico (Abal, Lozzia, Aguerri, Galibert, \& Attorresi, 2010). Además, se planea explorar la existencia de un factor de segundo orden.

\section{Referencias}

Abal, F. J. P., Lozzia, G. S., Aguerri, M. E., Galibert, M. S., \& Attorresi, H. F. (2010). La escasa aplicación de la teoría de respuesta al ítem en tests de ejecución típica. Revista Colombiana de Psicología, 19(1), 111-122. Recuperado de www.revistas.unal. edu.co/index.php/psicologia

Alessandri, G., Caprara, G. V., Eisenberg, N., \& Steca,
P. (2009). Reciprocal relations among self-efficacy beliefs and prosociality across time. Journal of Personality, 77(4), 1229-1259. doi: 10.1111/j.14676494.2009.00580.x

Auné, S., Abal, F., \& Attorresi, H. (2016a). Diseño y construcción de una Escala de Conducta Prosocial para Adultos. Revista Iberoamericana de Diagnóstico y Evaluación Psicológica, 42(2), 15-25. doi: 10.21865/RIDEP42_15

Auné, S., Abal, F., \& Attorresi, H. (2016b). Antagonismos entre concepciones de empatía y su relación con la conducta prosocial. Revista de Psicología, 17(2), 137-149. doi: 10.18050/revpsi.v17n2a7.2015

Auné, S., Blum, G. D., Abal, F., Lozzia, G., \& Attorresi, H. (2014). La conducta prosocial: Estado actual de la investigación. Perspectivas en Psicología, 11(2), 21-33.

Batson, C. D., \& Powell, A. (2003). Altruism and prosocial behavior. En M. Theodore \& L. Melvin (Eds.), Handbook of Psychology: Personality and Social Psychology, (5). Nueva York: John Wiley \& Sons, Inc. XIX.

Bearden, W. O., Sharma, S., \& Teel, J. E. (1982). Sample size effects on chi square and other statistics used in evaluating causal models. Journal of Marketing Research, 19(4), 425-430. doi: 10.2307/3151716

Beilin, H. (2013). The Development of Prosocial Behavior. N. Eisenberg (Ed.). Academic Press.

Buja, A., \& Eyuboglu, N. (1992). Remarks on parallel analysis. Multivariate Behavioral Research, 27(4), 509-540. doi: 10.1207/s15327906mbr2704 2

Caprara, G. V., Alessandri, A., Di Giunta, L., Panerai, L., \& Eisenberg, N. (2010). The contribution of agreeableness and self-efficacy beliefs to prosociality. European Journal of Personality, 24(1), 3655. doi: 10.1002/per.739

Caprara, G. V., Alessandri, G., \& Eisenberg, N. (2012). Prosociality: The contribution of traits, values, and self-efficacy beliefs. Journal of Personality and Social Psychology, 102(6), 1289-1303. doi: $10.1037 / \mathrm{a} 0025626$

Caprara, G. V., Capanna, C., Steca, P., \& Paciello, M. (2005). Misura e determinanti personali della prosocialità. Un approccio sociale cognitivo. Giornale Italiano di Psicologia, 32(2), 287-308. doi: 
$10.1421 / 20313$

Caprara, G. V., Steca, P., Zelli, A., \& Capanna, C. (2005). A new scale for measuring adults' prosocialness. European Journal of Psychological Assessment, 21(2), 77-89. doi: 10.1027/1015-5759.21.2.77

Carlo, G., \& Randall, B. A. (2001). Are all prosocial behaviors equal? A socioecological developmental conception of prosocial behavior. En F. Columbus (Ed.), Advances in Psychology Research, Vol. 2 (pp. 151-170). New York: Nova Science.

Carmines, E. G., \& Zeller, R. A. (1979). Reliability and validity assessment. doi: 10.4135/9781412985642

Castro-Solano, A. (2010). Ensayo: Psicología Positiva: ¿Una nueva forma de hacer psicología? Revista de Psicología, 6(11), 113-131. Recuperado de http:// bibliotecadigital.uca.edu.ar/greenstone/cgi-bin/ library.cgi? $\mathrm{e}=\mathrm{d}-00000-00---$ off-0Revistas--00-2---0-10-0---0---0direct-10---4-------0-11--10-esZz-1---20-about---00-3-1-00-0-0-01-1-0utfZz-8$00 \& \mathrm{a}=\mathrm{d} \& \mathrm{c}=$ Revistas $\& \mathrm{cl}=\mathrm{CL} 2.22$

DeCarlo, L. T. (1997). On the meaning and use of kurtosis. Psychological Methods, 2(3), 292-307. doi: 10.1037/1082-989X.2.3.292

Decety, J., \& Meyer, M. (2008). From emotion resonance to empathic understanding: A social developmental neuroscience account. Development and Psychopathology, 20(04), 1053-1080. doi: 10.1017/ S0954579408000503

DeVellis, R. F. (2003). Scale development. Theory and applications. Thousand Oaks, CA: Sage Publications.

Dunfield, K. A. (2014). A construct divided: Prosocial behavior as helping, sharing, and comforting subtypes. Frontiers in Psychology, 5, 958. doi: 10.3389/fpsyg.2014.00958

Dunfield, K., Kuhlmeier, V. A., O’Connell, L., \& Kelley, E. (2011). Examining the diversity of prosocial behavior: Helping, sharing, and comforting in infancy. Infancy, 16(3), 227-247. doi: 10.1111/j.15327078.2010.00041.x

Ferrando, P. J., \& Lorenzo-Seva, U. (2014). El análisis factorial exploratorio de los ítems: Algunas consideraciones adicionales. Anales de Psicología,
30(3). doi: 10.6018/analesps.30.3.199991

Garaigordobil, M., \& Berrueco, L. (2007). Autoconcepto en niños y niñas de 5 años: Relaciones con inteligencia, madurez neuropsicológica, creatividad, altruismo y empatía. Infancia y Aprendizaje, 30(4), 551-564. doi: 10.1174/021037007782334337

Garrido, L. E., Abad, F. J., \& Ponsoda, V. (2013). A new look at Horn's parallel analysis with ordinal variables. Psychological Methods, 18(4), 454-474. doi: 10.1037/a0030005

Glutting, J. J., Monaghan, M. C., Adams, W., \& Sheslow, D. (2002). Some psychometric properties of a system to measure ADHD among college students: Factor pattern, reliability, and one-year predictive validity. Measurement and Evaluation in Counseling and Development, 34, 194-208.

González-Portal, M. D. (2000). Conducta prosocial: Evaluación e Intervención. Madrid: Morata.

Hay, D. F., \& Cook, K. V. (2007). The transformation of prosocial behavior from infancy to childhood. En C. A. Brownell \& C. B. Kopp (Eds.), Socioemotional development in the toddler years: Transitions and transformations (pp. 100-131). New York: The Guilford Press.

Hendrickson, A. E., \& White, P. O. (1964). Promax: A quick method for rotation to oblique simple structure. British Journal of Statistical Psychology, 17(1), 65-70. doi: 10.1111/j.2044-8317.1964. tb00244.x

Hirschberger, G. (2006). Terror management and attributions of blame to innocent victims: Reconciling compassionate and defensive responses. Journal of Personality and Social Psychology, 91(5), 832844. doi: 10.1037/0022-3514.91.5.832

Holgado-Tello, F. P., Chacón-Moscoso, S., Barbero-García, I., \& Vila-Abad, E. (2010). Polychoric versus Pearson correlations in exploratory and confirmatory factor analysis of ordinal variables. Quality \& Quantity, 44(1), 153-166. doi: 10.1007/s11135008-9190-y

Horn, J. L. (1965). A rationale and test for the number of factors in factor analysis. Psychometrika, 30(2), 179-185. doi: 10.1007/BF02289447

Hu, L., \& Bentler, P. M. (1999). Cut off criteria for fit 
indexes in covariance structure analysis: Conventional criteria versus new alternatives. Structural Equation Modeling, 6(1), 1-55. doi: 10.1080/10705519909540118

Kelley, T. L. (1935). Essential traits of mental life. Cambridge, MA: Harvard University Press.

Lemos, V., \& Richaud de Minzi, M. C. (2014). Promotion of child prosocial behavior in the school context. En A. Castro-Solano (Ed.), Positive Psychology in Latin America. doi: 10.1007/978-94-017-9035-2

Lloret-Segura, S., Ferreres-Traver, A., Hernández-Baeza, A., \& Tomás-Marco, I. (2014). El análisis factorial exploratorio de los ítems: Una guía práctica, revisada y actualizada. Anales de Psicología, 30(3), 1151-1169. doi: 10.6018/analesps.30.3.199361

Lorenzo-Seva, U., \& Ferrando, P. J. (2013). FACTOR 9.2: A comprehensive program for fitting exploratory and semi confirmatory factor analysis and IRT models. Applied Psychological Measurement, 37(6), 497-498. doi: 10.1177/0146621613487794

Mardia, K. V. (1970). Measures of multivariate skewnees and kurtosis with applications. Biometrika, 57(3), 519-530. doi: 10.1093/biomet/57.3.519

Martínez-Arias, R. M., Hernández-Lloreda, M. V., \& Hernández-Lloreda, M. J. (2006). Psicometría. Madrid: Alianza Editorial.

Paulus, M., Kühn-Popp, N., Licata, M., Sodian, B., \& Meinhardt, J. (2013). Neural correlates of prosocial behavior in infancy: Different neurophysiological mechanisms support the emergence of helping and comforting. Neuroimage, 66, 522-530. doi: 10.1016/j.neuroimage.2012.10.041

Pérez, E., \& Medrano, L. (2014). Exploratory factor analysis: Conceptual and methodological basis. Revista Argentina de Ciencias del Comportamiento, 6(3), 71-80. Recuperado de https://revistas.unc. edu.ar/index.php/racc

Richaud de Minzi, M. C., Lemos, V., \& Mesurado, B., (2011). Relaciones entre la percepción que tienen los niños de los estilos de relación y de la empatía de los padres y la conducta prosocial en la niñez media y tardía. Avances en Psicología Latinoamericana, 29(2), 330-343. Recuperado de https:// revistas.urosario.edu.co/index.php/apl
Roche-Olivar, R. (1998). El uso educativo de la televisión como optimizadora de la prosocialidad. Psychosocial Intervention, 7(3), 363-378. Recuperado de http://www.psychosocial-intervention.org

Scourfield, J., John, B., Martin, N., \& McGuffin, P. (2004). The development of prosocial behavior in children and adolescents: A twin study. Journal of Child Psychology and Psychiatry, 45(5), 927-935. doi: 10.1111/j.1469-7610.2004.t01-1-00286.x

Tabachnick, B. G., \& Fidell, L. S. (2001). Using multivariate statistics. Boston: Ally and Bacon.

Vélez, L., \& Martínez-Lugo, M. (1995). El mundo del trabajo en Puerto Rico. Perfil motivacional de un grupo de trabajadores. Revista Latinoamericana de Psicología, 27(2), 283-304. Recuperado de http:// www.redalyc.org/articulo.oa?id=80527208

Warneken, F., \& Tomasello, M. (2006). Varieties of altruism in children and chimpanzees. Trends in Cognitive Sciences, 13(9), 397-402. doi: 10.1016/j. tics.2009.06.008

Warneken, F., \& Tomasello, M. (2009). The roots of human altruism. British Journal of Psychology, 100(3), 455-471. doi: 10.1348/000712608X379061 